\title{
Association between 2009 seasonal influenza vaccine and influenza-like illness during the 2009 pandemic: preliminary results of a large household transmission study in Western Australia
}

D Carcione ${ }^{1}$, C Giele ${ }^{1}$, L S Goggin ${ }^{1}$, K S Kwan ${ }^{1}$, D W Smith², G K Dowse ${ }^{1}$, D B Mak¹, P Effler (Paul.Effler@health.wa.gov.au) ${ }^{1}$

1. Communicable Disease Control Directorate, Department of Health, Perth, Western Australia, Australia

2. PathWest Laboratory Medicine WA, Nedlands, Western Australia, Australia

Citation style for this article:

Carcione D, Giele C, Goggin LS, Kwan KS, Smith DW, Dowse GK, Mak DB, Effler P. Association between 2009 seasonal influenza vaccine and influenza-like illness during the 2009 pandemic: preliminary results of a large household transmission study in Western Australia. Euro Surveill. 2010;15(28):pii=19616. Available online: http://www.eurosurveillance.org/ViewArticle.aspx?Articleld=19616

Article published on 15 July 2010

We conducted a prospective household transmission study to examine whether receipt of 2009 trivalent influenza vaccine (TIV) was associated with increased risk of influenza-like illness (ILI) among contacts of confirmed pandemic influenza $A\left(\mathrm{H}_{1} \mathrm{~N}_{1}\right) 2009$ patients. In the week following onset of pandemic illness in a household member, 46 (15\%) of 304 TIV-vaccinated contacts, and 174 (15\%) of 1,162 unvaccinated contacts developed ILI $(p=0.95)$. Receipt of 2009 TIV had no effect on one's risk of pandemic illness.

\section{Background}

Four recently described observational studies from Canada found that receipt of 2008-09 trivalent influenza vaccine (TIV) was associated with increased risk of pandemic influenza $\mathrm{A}\left(\mathrm{H}_{1} \mathrm{~N}_{1}\right)_{2009}$ during the spring and summer of 2009 [1,2]. Determining whether a seasonal influenza vaccine that does not contain pandemic viral antigens can affect one's risk of subsequent infection with the pandemic strain has important implications for public health, as well as our understanding of the immunopathogenesis of influenza infection. Household transmission studies are well-suited to examine this issue because prospective, active follow-up of household contacts can avoid many types of selection biases known to be associated with case-control studies [1].

We conducted a prospective household transmission study during the first ten weeks of the influenza season in Western Australia (29 May-7 August 2009) and examined whether prior vaccination with seasonal 2009 TIV increased the risk of developing influenzalike illness (ILI) among household contacts. Our results should be applicable to the experience in the northern hemisphere as the vaccine recommended by the World Health Organization for use during the 2009 southern hemisphere influenza season was identical to that used in the 2008-09 northern hemisphere influenza season (i.e. A/Brisbane/59/2007 (H1N1)-like virus, $A / B r i s b a n e / 10 / 2007\left(\mathrm{H}_{3} \mathrm{~N}_{2}\right)$-like virus, and $B /$ Florida/4/2006-like virus) $[3,4]$

\section{Methods}

Index patients were defined as the first symptomatic illness in the household with laboratory-confirmed pandemic influenza $A\left(\mathrm{H}_{1} \mathrm{~N}_{1}\right) 2009$ infection. Interviews with index patients, household contacts, or their carers, established a history of prior vaccination with 2009 TIV. Telephone follow-up with household contacts determined whether they had experienced an ILI in the period beginning at least one day after and within seven days of symptom onset in the index patient. ILI was defined as fever $>38^{\circ} \mathrm{C}$ (or a history of fever when the temperature was not taken) AND cough and/or sore throat. In addition, RT-PCR results on all household contacts with ILI who had a respiratory specimen collected via routine medical follow-up were reviewed. Included in this analysis were 595 households with 1,466 household contacts who had a known 2009 TIV vaccination history ( $90 \%$ of all household contacts). Chi-square tests were used assess statistical differences in proportions; $p$ values 0.05 were considered significant.

\section{Results}

Some 304 household contacts reported being vaccinated with 2009 TIV and 1,162 denied vaccination. The proportion of males and females in the vaccinated and unvaccinated cohorts was nearly identical, but the age group distributions differed significantly (Table). Nevertheless, among those with known age, the proportion of vaccinated and unvaccinated household contacts who were 18 years or older was similar $(63 \%$ and $66 \%$, respectively; $p=0.37$ ). Vaccinated household contacts were significantly more likely to report having diabetes or underlying heart, respiratory, or neurological disease (Table). The proportion of vaccinated and unvaccinated household contacts who received antiviral prophylaxis was $13 \%$ and $14 \%$, respectively. 
A total of $220(15 \%)$ of all household contacts developed ILI within seven days of the onset of illness in the index case; 27 of 29 household contacts with ILI who had a PCR specimen collected within 48 hours of symptom onset were positive for pandemic influenza $A\left(H_{1} N_{1}\right) 2009$ infection. Forty-six (15\%) of the 304 TIVvaccinated contacts and $174(15 \%)$ of the 1,162 unvaccinated contacts developed ILI $(p=0.95)$. When the analysis was restricted to the 941 household contacts aged 18 years or older, the proportion of contacts who developed ILI was $12 \%$ in the vaccinated cohort and $13 \%$ in the unvaccinated cohort $(\mathrm{p}=0.86)$. Prior vaccination with 2009 TIV was not associated with development of ILI among household contacts in logistic regression analyses that simultaneously controlled for age, sex, antiviral prophylaxis, diabetes, heart disease, respiratory disease, and neurological disease (odds ratio:1.0; $95 \%$ confidence interval: 0.7 to $1.5 ; p=0.95$ ).

\section{Discussion}

In Western Australia receipt of 2009 TIV was not associated with increased risk of developing ILI among household contacts of persons with confirmed pandemic influenza $A\left(\mathrm{H}_{1} \mathrm{~N}_{1}\right)$ 2009. Conversely, nor was there a protective effect of seasonal 2009 TIV vaccination.

Most investigations in settings outside Canada have found no relationship between 2008-09 TIV and pandemic influenza, but others have reported significant associations, both positive and negative. These studies have employed various methodologies including case-cohort, test-negative case-control, and crosssectional study designs [5-11]. The discrepant results across these studies most likely reflect differences in the study methods used, but actual variation in the effect of specific vaccines or disparities in the immunological background between populations cannot be discounted [1]. As the largest prospective study to report on this issue to date, our findings make an important contribution to the dialogue regarding the effect of seasonal TIV and the risk of developing pandemic influenza $A\left(\mathrm{H}_{1} \mathrm{~N}_{1}\right) 2009$.

Several limitations of our study should be noted. First, the outcome sought was clinical ILI and not laboratoryproven influenza. Only a small subset of household contacts who developed ILI were tested for influenza; however, a high proportion of the contacts tested by PCR within two days of developing ILI were confirmed as having pandemic influenza $A\left(\mathrm{H}_{1} \mathrm{~N}_{1}\right) 2009$ infection.

Second, vaccination histories were not verified through medical records. Most seasonal 2009 TIV vaccinations would have been administered between the months of March and June 2009, and interviews to determine vaccination status were conducted between late May and early August 2009. Whilst errors in recall may have occurred, it seems reasonable to assume that were this the case such errors would be similar among the cohort who later developed ILI, compared to those who did not.

A strength of our analysis is the ability to simultaneously control for age, antiviral prophylaxis and the presence of underlying medical conditions when examining the association of seasonal TIV and ILI in household contacts.

TABLE

Demographic characteristics of household contacts of pandemic influenza index cases, by 2009 TIV vaccination status, Western Australia $(n=1,466)$

\begin{tabular}{|c|c|c|c|}
\hline & \multicolumn{3}{|c|}{ Received 2009 TIV vaccine } \\
\hline & $\begin{array}{c}\text { Yes }(n=304) \\
n(\%)\end{array}$ & $\begin{array}{c}\text { No }(n=1,162) \\
n(\%)\end{array}$ & $\begin{array}{c}\text { Univariate } \\
\chi^{2}\end{array}$ \\
\hline \multicolumn{4}{|l|}{ Sex } \\
\hline Male & $150(49)$ & $597(51)$ & \multirow{2}{*}{$0.399^{\mathrm{a}}$} \\
\hline Female & $154(51)$ & $565(49)$ & \\
\hline \multicolumn{4}{|l|}{ Age group } \\
\hline o to 4 years & $44(14)$ & $105(9)$ & \multirow{5}{*}{$<0.001^{\mathrm{b}}$} \\
\hline 5 to 17 years & $65(21)$ & $287(25)$ & \\
\hline 18 to 50 years & $112(37)$ & $589(51)$ & \\
\hline$\geq 51$ years & $74(24)$ & 166 (14) & \\
\hline Unknown & $9(3)$ & $15(1)$ & \\
\hline \multicolumn{4}{|c|}{ Underlying medical conditions } \\
\hline Diabetes & $16(5)$ & $18(2)$ & $<0.005^{c}$ \\
\hline Heart disease & $13(4)$ & $19(2)$ & $<0.005$ \\
\hline Respiratory disease & $45(15)$ & $76(7)$ & $<0.005$ \\
\hline Neurological disease & $7(2)$ & $5(0)$ & $<0.005$ \\
\hline
\end{tabular}

TIV: trivalent influenza vaccine.

a Chi-square test for significant difference in sex distribution between vaccinated and unvaccinated household contacts.

${ }^{b}$ Chi-square test for significant difference in age group distribution between vaccinated and unvaccinated household contacts.

${ }^{c} \mathrm{Chi}$-square test for significant difference in specific underlying medical condition between vaccinated and unvaccinated household contacts. 
We agree with Janjua and colleagues $[12,13]$ that understanding the potential effect of seasonal TIV on pandemic illness has important ramifications and warrants rigorous investigations that provide sufficient information to assess their validity. The work presented here represents a sub-analysis of data collected as part of a comprehensive assessment of factors that influence the incidence of ILI in household contacts of persons with confirmed pandemic influenza $A\left(\mathrm{H}_{1} \mathrm{~N}_{1}\right) 2009$ infection; the full report containing additional detail on methods and participant characteristics is being prepared. In this interim analysis, we did not find an association, either positive or negative, between 2009 seasonal TIV and subsequent risk of ILI during the 2009 pandemic.

\section{References}

1. Skowronski DM, De Serres G, Crowcroft NS, Janjua NZ, Boulianne N, Hottes TS, et al. Association between the 2008og seasonal influenza vaccine and pandemic $\mathrm{H}_{1} \mathrm{~N}_{1}$ illness during Spring-Summer 2009: four observational studies from Canada. PLoS Med. 2010;7(4):e1000258.

2. Viboud C, Simonsen L. Does seasonal influenza vaccination increase the risk of illness with the $2009 \mathrm{~A} / \mathrm{H}_{1} \mathrm{~N}_{1}$ pandemic virus? PLoS Med. 2010;7(4):e1000259.

3. World Health Organization. Recommended composition of influenza virus vaccines for use in the 2008-2009 influenza season. [Accessed 1 July 2010]. Available from: http://www.who.int/csr/disease/influenza/recommended_ compositionFebo8FullReport.pdf

4. World Health Organization. Recommended composition of influenza virus vaccines for use in the 2009 southern hemisphere influenza season. [Accessed 1 July 2010]. Available from: http://www.who.int/csr/disease/ influenza/200809Recommendation.pdf

5. Kelly H, Grant K. Interim analysis of pandemic influenzA( $\left.\mathrm{H}_{1} \mathrm{~N}_{1}\right)$ 2009 in Australia: surveillance trends, age of infection and effectiveness of seasonal vaccination. Euro Surveill. 2009;14(31):pii=19288. Available from: http://www. eurosurveillance.org/ViewArticle.aspx?Articleld=19288

6. Garcia-Garcia L, Valdespino-Gomez JL, Lazcano-Ponce E, Jimenez-Corona A, Higuera-Iglesias A, Cruz-Hervert P, et al. Partial protection of seasonal trivalent inactivated vaccine against novel pandemic influenza $A / \mathrm{H}_{1} \mathrm{~N}_{1}$ 2009: case-control study in Mexico City. BMJ. 2009; 339:b3928.

7. Echevarria-Zuno S, Mejia-Arangure JM, Mar-Obeso AJ, GrajalesMuñiz C, Robles-Pérez E, González-León M, et al. Infection and deaths from influenza $\mathrm{A} \mathrm{H}_{1} \mathrm{~N}_{1}$ virus in Mexico: a retrospective analysis. Lancet. 2009;374(9707):2072-9.

8. Centers for Disease Control and Prevention (CDC). Effectiveness of 2008-09 trivalent influenza vaccine against 2009 pandemic influenza A(H1N1) - United States, May-June 2009. MMWR Morb Mortal Wkly Rep. 2009;58(44):1241-5.

9. Iuliano AD, Reed C, Guh A, Desai M, Dee DL, Kutty P, et al. Notes from the field: outbreak of 2009 pandemic influenza $A\left(\mathrm{H}_{1} \mathrm{~N}_{1}\right)$ virus at a large public university in Delaware, AprilMay 2009. Clin Infect Dis. 2009;49(12):1811-20.

10. Crum-Cianflone NF, Blair PJ, Faix D, Arnold J, Echols S, Sherman SS, et al. Clinical and epidemiologic characteristics of an outbreak of novel $\mathrm{H}_{1} \mathrm{~N}_{1}$ (swine origin) influenza A virus among United States military beneficiaries. Clin Infect Dis. 2009;49(12):1801-10.

11. Johns MC, Eick AA, Blazes DL, Lee SE, Perdue CL, Lipnick $R$, et al. Seasonal influenza vaccine and protection against pandemic $\left(\mathrm{H}_{1} \mathrm{~N}_{1}\right)$ 2009-associated illness among US military personnel. PLoS One. 2010;5(5):e10722

12. Janjua NZ, Skowronski DM, Hottes TS, De Serres G,

Crowcroft NS, Rosella LC. et al. Seasonal vaccine and $\mathrm{H}_{1} \mathrm{~N}_{1}$. Selection bias explains seasonal vaccine's protection. BMJ. 2009;339:b4972.

13. Janjua NZ, Skowronski DM, Hottes TS, De Serres G, Crowcroft NS, Rosella LC, et al. Seasonal vaccine effectiveness against pandemic $\mathrm{A} / \mathrm{H} 1 \mathrm{~N} 1$. Lancet. 2010;375(9717):801-2. 